OPEN ACCESS

Edited by:

Wayne William Hancock, University of Pennsylvania,

United States

Reviewed by: Heth Roderick Turnquist, University of Pittsburgh, United States Izabela Galvao,

Royal Prince Alfred Hospital, Australia

*Correspondence: Fernanda Degobbi Tenorio Quirino dos Santos Lopes fernandadtqs/@gmail.com

Specialty section:

This article was submitted to Immunological Tolerance and Regulation, a section of the journal

Frontiers in Immunology

Received: 29 October 2021 Accepted: 22 November 2021 Published: 09 December 2021

Citation: Lourenço JD, Ito JT, Martins MA, Tibério IFLC and Lopes FDTQS (2021)

Th17/Treg Imbalance in Chronic Obstructive Pulmonary Disease: Clinical and Experimental Evidence.

Front. Immunol. 12:804919. doi: 10.3389/fimmu.2021.804919

\section{Th17/Treg Imbalance in Chronic Obstructive Pulmonary Disease: Clinical and Experimental Evidence}

\author{
Juliana Dias Lourenço, Juliana Tiyaki Ito, Milton de Arruda Martins, Iolanda de Fátima \\ Lopes Calvo Tibério and Fernanda Degobbi Tenorio Quirino dos Santos Lopes* \\ Laboratory of Experimental Therapeutics (LIM-20), Department of Clinical Medicine, School of Medicine, University of \\ Sao Paulo, Sao Paulo, Brazil
}

The imbalance between pro- and anti-inflammatory immune responses mediated by Th17 and Treg cells is deeply involved in the development and progression of inflammation in chronic obstructive pulmonary disease (COPD). Several clinical and experimental studies have described the Th17/Treg imbalance in COPD progression. Due to its importance, many studies have also evaluated the effect of different treatments targeting Th17/Treg cells. However, discrepant results have been observed among different lung compartments, different COPD stages or local and systemic markers. Thus, the data must be carefully examined. In this context, this review explores and summarizes the recent outcomes of Th17/Treg imbalance in COPD development and progression in clinical, experimental and in vitro studies.

Keywords: Th17 cells (Th17), regulatory T cell (Treg), Th17/Treg imbalance, COPD, immune response

\section{INTRODUCTION}

Chronic obstructive pulmonary disease (COPD) is an inflammatory disease characterized by airway and/or alveolar abnormalities that lead to persistent airflow limitations and respiratory symptoms (1). According to the World Health Organization, COPD is currently the third leading cause of death, and it is predicted to remain so until 2030 (2). Although the primary cause of COPD is exposure to tobacco smoke, only approximately $25 \%$ of smokers develop the disease, which suggests that certain genetic, epigenetic, or host factors are involved in the amplified inflammatory response observed in COPD individuals (3).

COPD patients, especially when they have the disease in severe stages and during exacerbations, present systemic inflammation, which is associated with an accelerated decrease in lung function. This inflammation is characterized by increased circulating proinflammatory cytokines and chemokines, the levels of acute phase proteins, and abnormalities in circulating cells (4).

The inflammatory response in COPD involves both innate and adaptive immune responses (5, 6). T cell-mediated adaptive immunity is deeply involved in the regulation of airway inflammation during COPD development. During the developmental stage in the thymus, T lymphocytes differentiate into $\mathrm{CD} 8+\mathrm{T}$ and $\mathrm{CD} 4+\mathrm{T}$ cells. CD8 $+\mathrm{T}$ cells are the subtype of lymphocytes present in increased amounts in patients with COPD and they release proteolytic enzymes such as perforins and granzyme-B. These enzymes cause the death of structural cells by apoptosis or necrosis leading to the degradation of the extracellular matrix and remodelling, which results in the 
obstruction of small airways (6-8). In turn, CD4+ T cells can differentiate into different subtypes: T helper (Th)1, Th2, Th17 and regulatory $\mathrm{T}$ (Treg) cells $(3,5,9)$. The imbalance between proinflammatory and anti-inflammatory immune responses mediated by the different subsets of Th cells, such as Th17 and Treg cells, plays a pivotal role in the maintenance of inflammation and disease progression $(10,11)$.

For many years, CD4+ Th1 lymphocytes have been considered key players in the COPD inflammation process due to the high expression of cytokines related to the Th1 response, such as TNF- $\alpha$ (tumour necrosis factor- $\alpha$ ) during exacerbations and interferon- $\gamma$ (IFN- $\gamma$ ), in emphysematous patients (12-14). However, over the last decade, evidence from clinical and experimental studies has highlighted the importance of Th17 cells in promoting and maintaining the inflammatory process in COPD, as well as the failure of Treg cells to control this inflammatory process.

Th17 cells are characterized by the release of IL-17A, IL-17F and IL-22, which are associated with COPD progression and the exacerbation of alveolar destruction $(9,11,15,16)$. They are found mainly in the bronchial mucosa and express the transcription factor $R O R \gamma t$ as a specific marker $(15,17)$. In contrast, Treg cells are responsible for the regulation of immune responses by suppressing inflammation and autoimmunity through the release of anti-inflammatory cytokines, such as IL-10 and transforming growth factor- $\beta$ (TGF- $\beta)(5,9)$. These suppressive functions are dependent on the expression of the transcription factor Foxp3 (18). IL-10 is an interleukin produced by macrophages, dendritic cells, B cells, CD8+ T cells and several CD4+ T subtypes, and is mainly known for its anti-inflammatory effects (19-22). These cells are recognized as limiting the production of proinflammatory cytokines and chemokines such as IL- $1 \beta,-6,-12,-18$, TNF- $\alpha$, and MIP-1, among others. In addition, in the absence of IL-10, the IL-23 levels may increase, leading to their differentiation into Th17 cells $(11,20,23,24)$.

Although clinical and experimental studies have described the Th17/Treg imbalance in COPD progression (10, 16, 25-35), there are discrepant results when comparing these studies. Moreover, these discrepancies are also observed when analyses are performed in different lung compartments or when comparing samples from lungs to peripheral blood.

In this context, this review intends to explore and summarize the recent outcomes of Th17/Treg imbalance in COPD development and progression in clinical, experimental and in vitro studies.

\section{TH17/TREG IN CLINICAL STUDIES}

\subsection{Local Response}

The discussions around the Th17/Treg imbalance in COPD gained strength with the studies of Lee et al. (2007) and Cosio et al. $(2009)(6,12)$. Lee and colleagues (12) proposed that a reduction in the number of Treg cells was associated with the progression of COPD, causing an imbalance between the proand anti-inflammatory responses. In this study, a reduction in
Foxp3 mRNA expression and IL-10 secretion was observed in the tissue of patients with COPD, suggesting a lower regulatory response in those patients.

From these observations, it has been proposed that exposure to cigarette smoke induces innate immune cells to secrete proteolytic enzymes, releasing fragments of elastin that can be recognized as autoantigens and initiate a process of autoimmunity mediated by $\mathrm{T}$ and $\mathrm{B}$ cells against self-elastin fragments. In addition, the authors proposed that Treg cells are essential to control this inflammatory response in an attempt to suppress the response to autoantigens. In accordance, Cosio et al. (2009) (6) proposed that the progression of COPD and the severity of this disease are determined both by the ability of dendritic cells to stimulate $\mathrm{T}$ cells and by the immunoregulatory actions dependent on Tregs. They suggested that a serious failure in this control of the inflammatory process mediated by Tregs results in advanced stages of COPD.

\subsubsection{Th17/Treg Immune Response Markers}

Proinflammatory markers that characterize the immune response mediated by Th17 cells are usually increased in COPD patients. The IL-17A and IL-17F cytokines are predominantly released by Th17 cells, which are linked to neutrophilic inflammation, as IL-17 induces airway epithelial cells to release neutrophil chemotactic chemokines such as CXCchemokine ligand 1 (CXCL1) and CXCL8, attracting neutrophils into the airways (36). Di Stefano et al. (2009) evaluated Th17related cytokines (IL-17A, IL-22 and IL-23) in bronchial biopsies of patients with stable COPD (15). Although IL-17A is considered the main effector cytokine of Th17 cells, an increase in IL-17-positive cells was detected only in the bronchial submucosa of individuals with COPD and healthy smokers. The authors showed no significant difference in IL-17Apositive cells in the bronchial epithelium or in the gene expression of this interleukin between smokers with or without COPD or among different stages of COPD (15).

Zhang et al. (2013) evaluated the levels of IL-17 and IL-22 in the sputum of nonsmokers, healthy smokers and patients at 4 different COPD GOLD stages (16). The IL-17A and IL-22 levels were increased in COPD stages III and IV compared to the other groups, and sputum IL-17A levels in COPD patients were positively correlated with sputum neutrophils, enhancing the inflammatory process (16). In agreement with these results, Roos et al. (2015) observed increased IL-17A levels in the bronchoalveolar lavage fluid (BALF) of patients with COPD during acute exacerbations induced by Haemophilus infuenzae (NTHi) (37).

Regarding the anti-inflammatory process mediated by the Treg response, decreased numbers of Treg cells in the bronchial epithelium were reported in patients with severe and very severe COPD compared to those with mild and moderate COPD and healthy smokers (38). These results demonstrated that lower levels of Treg cells in the blood and lungs were observed only in severe COPD stages. In addition, Chu et al. (2011) demonstrated decreased Foxp3+ cells and gene and protein expression in tissue samples from moderate and severe COPD patients compared to healthy smokers and never smokers (29). 
Contrasting results were observed when evaluating the Treg response in BALF. Smyth et al. (2007) observed an increase in the number of Treg+ cells in the BALF of COPD and healthy smokers compared with nonsmokers (39). However, the authors observed that those cells expressed lower levels of $\mathrm{CD} 27+$, a marker of more potent regulatory activity, which suggests weaker $\mathrm{T}$ regulatory activity in BALF than peripheral blood mononuclear cells (39). More recently, Ström et al. (2020) compared the proportion of Tregs with regulatory function (Foxp3+/CD4+CD25bright) among COPD smokers, healthy smokers, and control subjects (40). The authors also found that, despite no significant difference found among the groups, the proportions of Tregs with regulatory function were significantly lower in COPD subjects with a rapid decline in lung function than in those with a nonrapid decline (40).

Zheng et al. (2018) evaluated the Th17/Treg imbalance in tissue samples of COPD stage I and II patients and compared them to healthy smokers and controls (41). They observed a progressive increase in Th17 cells and a decrease in Treg cells in the COPD group, as measured by both immunohistochemistry and flow cytometry (41). In a previous study, our group demonstrated the Th17/Treg imbalance in tissue samples from COPD subjects (42). In this study, an increase in IL-17+ cells was observed in both the COPD and healthy smoker groups, whereas Treg (Foxp3+) and IL-10+ cell numbers were decreased in the small airways of obstructed smokers compared with healthy smokers and control subjects. In contrast, the authors also noted an increase in Treg cells in lymphoid tissues (42).

Similar results were observed by Plumb et al. (2009), in which the authors found increased Treg cells in the follicles of moderate COPD patients compared with those in the follicles of smokers and nonsmokers (43). Additionally, Isajevs et al. (2009) demonstrated an upregulation of Foxp3+ expression in the large airways of COPD patients compared to controls, although a downregulation was observed in the small airways (44).

\subsubsection{The Importance of Intracellular Signalling in Th17/Treg Differentiation}

$\mathrm{CD} 4+\mathrm{T}$ cell subset differentiation is dependent on antigen (Ag) nature, the type of antigen presenting cells (APCs), and the cytokines present in the microenvironment. Within the APCs, there are the dendritic cells (DCs), which are the unique one with capacity to activate $\mathrm{T}$ cells and initiate primary immune responses in lymph nodes (45). Therefore, DC derived signals can promote $\mathrm{CD} 4+\mathrm{T}$ helper cell differentiation and CD8+ cytotoxicity (5). In COPD, Shan et al. (2009) showed that human lung DCs isolated from subjects with advanced emphysema could differentiate CD4+ T cells into distinct effector subsets (46). The authors demonstrated that total CD4+ T cells from PBMC that were cocultured with CD1a+ from lungs of subjects with advanced emphysema, but not control subjects, secreted higher amounts of IFN- $\gamma$ and IL-17A. $\mathrm{CD} 1 \mathrm{a}+$ is a marker of Langerhans cells, a subtype of dendritic cell with antigen-presenting functions. Moreover, CD4+ T cells cultured with CD1a+ DCs from control, but not emphysema, differentiated into Treg cells, evidenced by an up-regulation of Foxp3 expression (46).

The cytokines present in the microenvironment are, however, the most important element on the CD4+ T cell subset differentiation. Cytokines are recognized by their cell surface receptors, which activate the Janus kinase-signal transducer and activator of transcription (JAK-STAT) pathway. JAKs are tyrosine kinases that bind to the cytoplasmic regions of type I and II cytokine receptors that, once activated, lead to the recruitment and phosphorylation of STAT proteins (47). Th17 differentiation is induced by the expression of $R O R \gamma t$, which is dependent on the activation of STAT3 in the presence of IL-6, IL-21 and IL-23. In contrast, STAT5 can promote Treg skewing by regulating Foxp3 expression in the presence of TGF- $\beta$ and IL2 (47-49). Despite the importance of STAT proteins in regulating immunological responses, including Th17 and Treg skewing, only a few studies have evaluated them in COPD.

An increase in ROR $\gamma \mathrm{t}$ gene and protein expression was observed in a study conducted on tissue samples from patients with moderate and severe COPD who underwent pulmonary resection for peripheral carcinoma (29). Yew-Booth et al. (2015) demonstrated that stage III and IV COPD patients present with an increase in phosphorylated STAT3 protein in the lungs compared to nonobstructed smokers and never smokers, although no difference was observed in STAT5 expression (50). In a previous study by our group, ROR $\gamma t$ and STAT3 gene expression was also found to be increased in the lungs of mild and moderate COPD patients compared to those of healthy smokers (27). These findings were accompanied by increased $I L$ 6 and TGF- $\beta$ gene expression and IL- 6 levels, demonstrating early Th17 skewing. In this study, despite the increase in Foxp3 gene expression in the lungs, no difference was observed in STAT5 and IL-10 gene expression or IL-10 levels (27).

Within the process of cellular homeostasis, there are also the important role played by the Suppressors of Cytokine Signalling (SOCS) proteins. They are small intracellular proteins that are responsible for inhibiting the activation of STATs, block their recruitment to the cytokine receptor or inhibit their phosphorylation by JAKs (51). Thus, SOCS proteins are an essential piece on the regulation of $\mathrm{CD} 4+\mathrm{T}$ cells skewing into their subtypes. Springer et al. (2013) evaluated the gene expression of SOCS3, a well-defined STAT3 inhibitor and therefore affects Th17 skewing (52), in bronchial mucosa from COPD patients (53). The authors found that SOCS3 expression was downregulated compared to that in nonsmoker control subjects, consistent with a Th17 response (53). In turn, SOCS1 can promote Th17 differentiation by regulating SOCS3 and inhibiting IFN- $\gamma$ release (51). In addition, SOCS1 are related to the integrity and function of Treg cells, through the maintenance of Foxp3 expression (54). In patients with stage II COPD, decreased SOCS1 expression was observed both in the bronchial mucosa and in alveolar bronchoalveolar lavage macrophages (55).

It is important to consider the limitations and difficulties when studying some molecular and immunologic COPD aspects in humans, especially in lung tissue samples. Usually, COPD 
patients who present with constant exacerbations or who are in severe stages of the disease are not often submitted to procedures where lung tissue samples can be obtained. In contrast, healthy smokers or nonsmokers are usually undergo these procedures due to a confirmed tumour or a suspicion of neoplastic disease, which can be a limitation of some studies. Moreover, comparing tissue findings with those of BALF and peripheral blood can be hard to correlate because most studies do not obtain these samples from the same individual. Thus, the data must be carefully examined since the results from studies of one compartment may not be applicable to others.

\subsection{Systemic Response}

COPD patients who present with persistent systemic inflammation usually have worsening clinical outcomes, which negatively impacts their comorbidities, including an increase in the frequency of exacerbations and the risk of death (56). Whether systemic inflammation occurs as a result of the release of inflammatory mediators ("spill over") from the lungs, where the inflammation begins, or whether it is the result of some comorbidity that then affects the lungs is still uncertain $(4,57,58)$.

Many studies that have evaluated systemic Th17 response markers have revealed their increased levels in advanced COPD stages. Wang et al. (2015) evaluated peripheral blood samples from individuals who had never smoked, smokers without obstruction and individuals with moderate (stages II and III) and severe (stage IV) COPD (10). Patients with moderate and severe COPD had a higher frequency of Th17 cells, elevated levels of ROR $\gamma t$ mRNA expression and increased serum levels of IL-17A, IL-6, IL-21, IL-22 and IL-23. The authors also showed a lower frequency of Treg cells, and decreased Foxp3 mRNA expression and serum level of IL-10 in those patients. Additionally, it was demonstrated that the increase in the Th17/Treg ratio was negatively correlated with the worsening of lung function in those patients (10). In accordance, Silva et al. (2018) observed that patients with stage III COPD have more circulating IL- 6 and less IL-10 than individuals with stage I and II disease (31).

We observed similar results evaluating both plasma and isolated total leucocytes from the peripheral blood of nonobstructive smokers (NOS), patients with COPD stages I and II, and COPD stages III and IV (27). Increased STAT3, $R O R \gamma t, I L-6$ and TGF- $\beta$ gene expression were observed in white blood cells from the COPD III and IV patients compared to those in stages I and II and NOS. Although STAT5 was increased in the COPD III and IV group, there was a decrease in Foxp3 expression and IL-10 levels in the COPD I and II and COPD III and IV groups, respectively, which was different from the tissue findings (27).

Contrasting results were shown by Vargas-Rojas et al. (2011). They demonstrated a progressive increase in both Th17 and Treg cell subsets in peripheral blood mononuclear cells (PBMCs) from COPD patients and smokers without COPD compared to healthy subjects (59). The Treg increase was attributed to longterm exposure to cigarette smoke but not airway obstruction. Additionally, the authors demonstrated a negative correlation between Th17 cells and $\mathrm{FEV}_{1}$ and $\mathrm{FEV}_{1} / \mathrm{FVC}$ values. In accordance, $\mathrm{Xu}$ et al. (2019) demonstrated a negative correlation between the percentages of circulating Th17 cells, which was found increased in COPD patients compared to smokers and never smokers, and FEV1\% predicted values (60). Moreover, Zhang et al. (2013) found that serum levels of IL-17A progressively increased within COPD stages, although no difference was observed in the serum IL-10 levels between COPD patients and healthy smokers (16).

\subsubsection{Th17/Treg Imbalance in Stable and Exacerbated COPD Patients}

Clinical studies have been performed to better understand the Th17/Treg imbalance during COPD exacerbations, and most of them showed increased levels of both Th17 and Treg response markers.

Li et al. (2014) observed an increased Treg cell proportion in the peripheral blood of both acute exacerbation and stable COPD individuals compared to healthy smokers and an increased Th17 cell proportion only in the exacerbated COPD group (30). However, the authors observed a negative correlation between Th17 and Treg responses in both acute exacerbations and stable COPD. In contrast, Jin et al. (2014) (28) demonstrated an increase in inflammatory (IL-17 and TNF- $\alpha$ ) and antiinflammatory (IL-10 and TGF- $\beta$ ) cytokines in patients with exacerbated COPD, in addition to an increase in Treg cells. They suggested that although the Treg/IL-17 ratio showed normal values, Treg cells were insufficient to suppress the increase in mediators associated with inflammation.

Moreover, Zheng et al. (2018) found a progressive increase in Th17 cells in PBMCs of healthy smokers, individuals with stable COPD and those with exacerbated COPD compared to healthy nonsmokers (41). The authors also demonstrated a progressive decrease in Treg cells in those groups, evidence of a Th17/Treg imbalance. These results were in accordance with the lung tissue findings observed by the authors in the same study. However, PBMC samples were not obtained from the same subjects from which the lung tissue samples were collected (41). In fact, only a few studies have evaluated samples from lungs and peripheral blood obtained from the same patient $(27,38)$, which can be a limiting factor when studying human samples. Table 1 summarizes the main findings regarding the Th17/Treg imbalance in clinical studies, divided by inflammatory marker and by systemic and local responses.

\section{TH17/TREG IN EXPERIMENTAL MODELS OF COPD}

\subsection{Local and Systemic Responses}

Considering the difficulties of obtaining lung samples from patients, experimental studies are useful for studying different lung compartments and systemic responses in the same individual. Thus, we described the Th17/Treg imbalance in experimental models of COPD for both local and systemic responses in a single topic. Nevertheless, findings obtained 
TABLE 1 | Summary of the main findings regarding Th17/Treg imbalance in clinical studies.

\section{CLINICAL STUDIES}

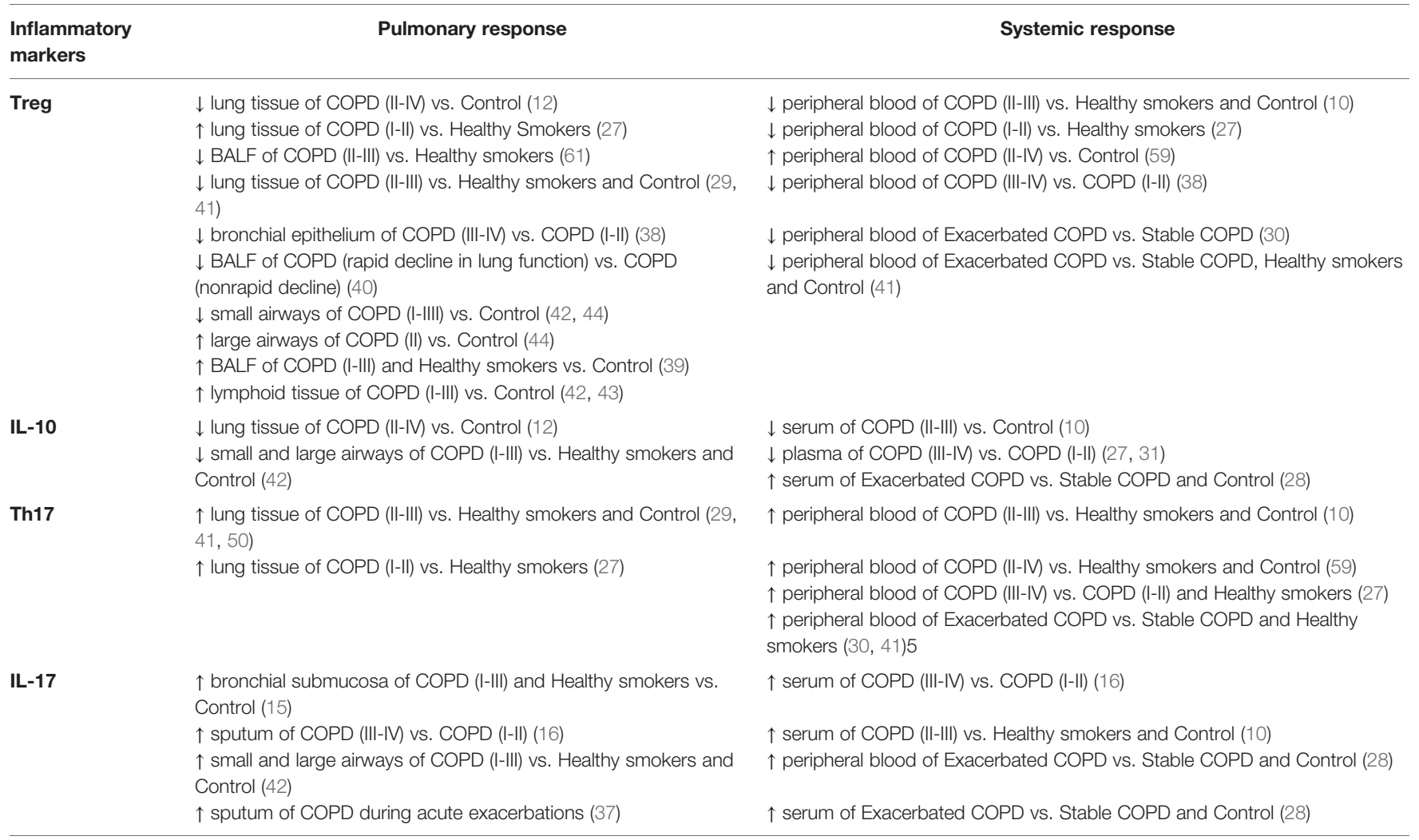

Data are presented divided by inflammatory marker and by the evaluated compartment (pulmonary and systemic response). Treg, regulatory T cell; IL-10, interleukin-10; Th17, Thelper-17, IL-17, interleukin-17; COPD, chronic obstructive pulmonary disease; vs., versus; BALF, bronchoalveolar lavage fluid; $\uparrow$ : increased; $\downarrow$ : decreased.

from animal models are described in Table 2, discriminated by each different compartment.

Despite the evidence of a Th17/Treg imbalance and the worsening of lung function in clinical studies, some pathophysiological mechanisms can only be evaluated using animal models. In animal models, it is possible to perform temporal evaluations, which are crucial to obtain and improve the understanding of pro- and anti-inflammatory immune response changes during COPD development, including at different time points during disease progression.

In a temporal analysis, Wang et al. (2012) evaluated the lung tissue, BALF and peripheral blood of mice exposed to cigarette smoke (CS) for 4 and 24 weeks (32). The authors showed that mice chronically exposed to CS presented an increased Th17 prevalence (CD4+IL-17+/CD4+ T cells) in lung tissue and peripheral blood and increased $R O R \gamma t$ mRNA expression in the lungs compared with control and sub-acute CS exposed mice. Moreover, chronic exposure led to increased levels of Th17-related cytokines (IL-17A, IL-6, IL-23) in BALF and serum. In contrast, the Treg response was first increased in subacute CS exposure group compared to control mice and then it decreased in the chronic exposure group. Additionally, the Treg prevalence (CD4+CD25+ Foxp3+/CD4+ T cells) in both the lung tissue and peripheral blood and Foxp 3 mRNA expression in the lungs and IL-10 in serum and BALF were significantly decreased in mice chronically exposed to CS (32).
Duan et al. (2016) showed similar results in mice exposed to CS for 12 and 24 weeks (33). The authors observed increased frequencies of Th17 (CD4+IL-17+) cells, ROR $\gamma t$ mRNA expression and IL-6, IL-17, and TGF- $\beta 1$ levels in the lungs of both groups exposed to CS compared to the control group, with higher values in the 24-week group. These findings were accompanied by a progressive decrease in Treg (CD4+Foxp3+) frequencies and Foxp3 and IL-10 expression in the CS-exposed groups. Additionally, the frequencies of Tregs were negatively correlated with Th17 cells (33). These findings were consistent with a previous study from the same group, in which it was demonstrated that Th17 cell, ROR $\gamma t$ and $I L-17 A$ mRNA expression were increased in the lungs of CS-exposed mice, even after 12 weeks of smoke exposure cessation (63).

In a previous study, we showed in temporal analyses that mice exposed to CS for 1,3 and 6 months presented an increase in IL-17+ cells in the peribroncovascular area after the sixth month of exposure (25). However, reductions in IL$10+$, TGF- $\beta+$ and Treg cell numbers were observed since the initial events of COPD development and were associated with decreases in lung function. Although the numbers of Treg cells in mice exposed to CS returned to numbers similar to those in the control group in later stages, the reductions in IL$10+$ and TGF- $\beta+$ cell numbers persisted until the end of the protocol, suggesting that despite the amount of Treg cells 
TABLE 2 | Summary of the main findings regarding Th17/Treg imbalance in experimental studies.

\section{EXPERIMENTAL STUDIES}

\begin{tabular}{|c|c|c|}
\hline $\begin{array}{l}\text { Inflammatory } \\
\text { markers }\end{array}$ & Pulmonary response & Systemic response \\
\hline Treg & $\begin{array}{l}\uparrow \text { lung tissue of Sub-acute CS exposed mice vs. Control (32) } \\
\downarrow \text { lung tissue of Chronic CS exposed mice vs. Control }(32,33) \\
\downarrow \text { peribronchovascular areas of Sub-acute CS exposed mice vs. Control (25) } \\
\uparrow \text { lung parenchyma of CS and LPS exposed mice vs. Control (35) } \\
\downarrow \text { lung tissue of CS and NTHi exposed mice vs. CS exposed mice (34) }\end{array}$ & $\begin{array}{l}\uparrow \text { peripheral blood of Sub-acute CS exposed mice vs. Control (32) } \\
\downarrow \text { peripheral blood of Chronic CS exposed mice vs. Sub-acute CS } \\
\text { exposed mice and Control (32) }\end{array}$ \\
\hline IL-17 & $\begin{array}{l}\uparrow \text { BALF of Chronic CS exposed mice vs. Sub-acute CS exposed mice } \\
\text { and Control }(32,62) \\
\uparrow \text { lung tissue and BALF of Chronic CS exposed mice vs. Control }(62) \\
\uparrow \text { lung tissue of Chronic and Sub-acute CS exposed mice vs. Control }(33,62) \\
\uparrow \text { lung tissue of Chronic CS exposed mice vs. Sub-acute CS exposed } \\
\text { mice and Control }(33,62) \\
\uparrow \text { peribronchovascular areas of Chronic CS exposed mice vs. Control }(25,26) \\
\uparrow \text { lung parenchyma of CS and LPS exposed mice vs. Control }(35) \\
\uparrow \text { lung tissue of CS and NTHi exposed mice vs. CS exposed mice and } \\
\text { Control (34) }\end{array}$ & $\begin{array}{l}\uparrow \text { serum of Chronic CS exposed mice vs. Sub-acute CS exposed mice } \\
\text { and Control (32) }\end{array}$ \\
\hline
\end{tabular}

Data are presented divided by inflammatory marker and by the evaluated compartment (pulmonary and systemic response). Treg, regulatory T cell; IL-10, interleukin-10; Th17, Thelper-17, IL-17, interleukin-17; CS, cigarette smoke; vs., versus; BALF, bronchoalveolar lavage fluid; LPS, lipopolysaccharide; NTHi, Haemophilus influenzae; $\uparrow:$ increased; $\downarrow:$ decreased.

present in the lung tissue, there is an impairment of immunosuppressive activity (25).

Thus, in another study, we demonstrated decreases in intracellular signalling for Treg cell differentiation and IL-10 release in peribronchovascular areas of the lung that occurred prior to Th17 signalling in mice exposed for 3 and 6 months to CS (26). A decrease in STAT5+ and pSTAT5+ cells and IL-10 expression was demonstrated at the 3rd month of exposure, whereas Th17 skewing was detected only at the 6th month with increased STAT3+ and pSTAT3+ cell numbers and IL-17 and IL-6 levels. We also demonstrated an increase in SOCS1+ density from the 3rd month of exposure, concomitantly with a decrease in SOCS3+ density (26). In agreement, Zhou et al. (2015) evaluated the Th17 response in mice exposed to CS for 2, 8, 12 and 24 weeks (62). They observed increased IL-17 levels in the lungs since week 2 that persisted until week 24, increased Th17 cells (characterized as CD3+CD8-IL-17A+) in the lungs since week 8 , and increased IL-17 levels in the BALF of groups exposed to CS for 12 and 24 weeks. Interestingly, RORc and STAT3 mRNA expression was increased only in mice exposed to CS for 8 and 12 weeks, while increased $I L-6$ mRNA was also observed, but not in the 24-week-exposed group (62). These findings corroborate Ruwanpura et al. (2014), who also demonstrated in mice that IL- 6 induced STAT3 activation, leading to Th17 cell differentiation during the inflammatory process (64).

\subsection{Animal Models of COPD Exacerbation}

Th17/Treg imbalance was also demonstrated by our research group to lead to an exacerbation of the inflammatory process in an animal model of CS exposure associated with lipopolysaccharide challenge (35). We showed that mice exposed to CS and associated with LPS instillation showed an increase in the inflammatory mediators STAT3+, pSTAT3+ and IL-17+, as well as the anti-inflammatory mediators STAT5+, pSTAT5+ and Treg (Foxp3+). However, despite the increase in Treg cells, the decrease in IL-10+ cell density observed in the CS/ LPS group suggests that the failure of cytokine release plays a fundamental role in exacerbation of the inflammatory response (35).

In another experimental model of COPD exacerbation, female mice were exposed to CS and then were administered Haemophilus influenzae (NTHi) (34). A progressive increase in IL-17 levels in the serum and IL-6 levels and ROR $\gamma$ t gene and protein expression in the lungs of the CS, NTHi and CS+NTHi 
groups compared to the control group was found. However, Foxp3 gene expression was decreased in the CS+NTHi group, although no differences were observed among the groups when analysing the Foxp3 protein levels using IHQ and WB. The authors concluded that NHTi infection impaired the antiinflammatory Treg balance, which combined with the strong effect of Th17 cells in the lungs leads to a Th17/Treg imbalance and AECOPD development (34). In accordance, Roos et al. (2015) previously reported that CS-exposed mice infected with NTHi were associated with an induction of IL-17A in the lungs followed by neutrophil recruitment, reinforcing the strength of the inflammatory process mediated by Th17 cells in NTHiassociated AECOPD (37).

The comparison of previous studies using human lung samples $(29,42)$ with the lungs of CS-exposed mice shows that the main difference in the inflammatory signal is the lung compartment in which they are detected. It appears that in humans, the inflammatory infiltrate is mainly observed in the airways between the respiratory epithelium and the adventitia (15, 42, 65), whereas in a CS-exposed animal model, inflammatory infiltrates are observed mostly in peribronchovascular areas between the airways and adjacent blood vessels $(25,26,35)$. This difference likely occurs due to the specialized capillaries in this area, which allow for the rapid release of leukocytes and therefore results in oedema and inflammatory infiltration (66).

\section{TREATMENTS TARGETING TH17/TREG CELLS IN COPD AND IN VITRO STUDIES}

The most common treatment of COPD patients is the use of longacting $\beta 2$-agonists and anticholinergic drugs, which improves the clinical manifestations of airway obstruction. Moreover, during exacerbations or depending on the COPD stage, the combination of anti-inflammatory treatment with inhaled corticosteroids (ICS) and one or two long-acting bronchodilators (1), is also used, which is known to reduce the number of CD8+ T lymphocytes in the bronchial mucosa of those patients (67). However, the antiinflammatory effects on other adaptive and innate immune cells were much lower than those observed in asthma $(67,68)$. Thus, studies that attempt to identify new treatment targets are necessary to better understand the imbalance between pro- and antiinflammatory responses in COPD. In this context, in vitro and experimental studies are useful (69).

Profita et al. (2014) demonstrated in PBMCs cultured cells that treatment with tiotropium spiriva, an anticholinergic drug, and olodaterol, a long-acting $\beta 2$-agonist, can control systemic inflammation through the Th17/Treg balance (70). The authors found that T-cells from COPD patients had higher levels of the neurotransmitter of the cholinergic system acetylcholine (Ach) coexpression with IL-17A, IL-22 and ROR $\gamma$ t and that ACh might promote the increased levels of Th17 cells in systemic inflammation. Additionally, treatment with tiotropium and olodaterol reduced the percentage of T cells coexpressing AChIL17A, AChIL-22, and AChROR $\gamma$ t while increasing the Foxp3 expression in COPD patients (70).
Using antibiotics, Tan et al. (2016) evaluated treatment with erythromycin for 6 and 12 months in induced sputum and peripheral blood of COPD stage II-IV patients (71). The authors showed that the treatment was effective in decreasing the IL-17 and IL-23 levels in both the sputum and serum of individuals treated for 12 months compared with the placebotreated group and 6-month-treated group (71).

In accordance, mice that received elastin peptides (EP) intranasally, treatment with erythromycin was sufficient to reduce the IL-17 and IL- 6 levels in both the serum and BALF compared to the EP group (72). Additionally, treatment with erythromycin modulated the $\mathrm{CD} 4+\mathrm{T}$ cell responses by reducing the Th17 cells (IL-17+CD4 $\mathrm{T}+$ frequency and $I L-17$ mRNA) and increasing the Treg response (FOXP3+CD4+ CD25+ frequency and Foxp3 mRNA) in the peripheral blood, spleen, and lungs (72). Additionally, Liu et al. (2020) treated cigarette smoke extract (CSE)-exposed dendritic cells (DCs) with erythromycin and demonstrated that treatment suppressed the CSE-exposed DC-mediated polarization of CD4+ T cells into Th17 cells (73).

Treatment with N-Acetylcysteine (NAC), an antioxidant that works as a mucolytic agent, was also effective on restore the Th17/Treg imbalance in COPD patients (74). In this study, the oral administration of NAC for 6 months led to a decrease in Th17 cells and increase in Treg cells of PBMC from COPD treated patients compared to the nontreated group. These findings were accompanied with decreased serum levels of inflammatory cytokines such as IL-17 and IL-1 $\beta$ and increased of the anti-inflammatory IL-10 level (74). In addition, the authors demonstrated that NAC regulated Th17/Treg balance through Hypoxia Inducible Factor- $1 \alpha(\mathrm{HIF}-1 \alpha)$ pathway, which is molecule that can upregulate the IL-17 expression through ROR $\gamma t$ and in addition to degrade Foxp3 $(74,75)$. The COPD NAC-treated group showed significantly decreased HIF1- $\alpha$ expression of Th17 and Treg cells isolated from peripheral blood (74).

More recently, the efficiency of treatment with anti-IL-17 has been demonstrated to improve the inflammation observed in asthma $(76,77)$, asthma exacerbated by LPS $(78,79)$ and LPSinduced lung injury (80). In an elastase-induced model of emphysema, it was also demonstrated that both preventive and therapeutic treatment with anti-IL17 improved the evaluated inflammatory parameters in mice, including specific markers for the Th17 response (81).

Considering the importance of corticosteroids, Nasreen et al. (2014) demonstrated that treatment with fluticasone furoate and mometasone furoate, two glucocorticoids usually recommended for the treatment of allergic rhinitis and other inflammatory diseases, in epithelial cell cultures exposed to cigarette smoke, promoted an increase in SOCS3 (82). Considering that SOCS3 can prevent Th17 skewing through STAT3 inhibition, the authors suggested a new mechanism for this drug to induce an anti-inflammatory effect. In addition, both treatments were able to restore the inhibited SOCS3 expression in the airway epithelium of CS-exposed mice (82). In contrast, treatment with budesonide (ICS) and formoterol 
(long-acting $\beta 2$-agonist bronchodilator), both commonly used for COPD treatment, was ineffective in restoring the loss of CD4+CD25highCD127- cells, a Treg population with highly suppressive functions isolated from PBMCs of both current and former COPD smokers (83).

\section{FINAL CONSIDERATIONS AND FUTURE DIRECTIONS}

It has been demonstrated that in both clinical and experimental studies, an increased Th17 response is involved in the progression of obstruction in COPD, even when comparing different lung compartments or systemic and local responses. These changes are frequently observed in more advanced stages of disease progression in experimental studies, while clinical studies demonstrate Th17 skewing since early COPD stages.

On the other hand, discrepant results have been observed, especially in studies that have described the immune response mediated by Treg cells. Depending on the compartment analysed, or if lung and blood samples are collected from the same individual, differences are observed in Treg amounts and/ or function. Nevertheless, most studies are in agreement with the IL-10 analysis, describing the decrease in IL-10 release during COPD development and progression. Figure 1 elucidates the general findings about Th17/Treg imbalance in clinical and experimental studies, in both stable and exacerbated disease, considering not only the cell amounts but also the cells function and the release of related cytokines.

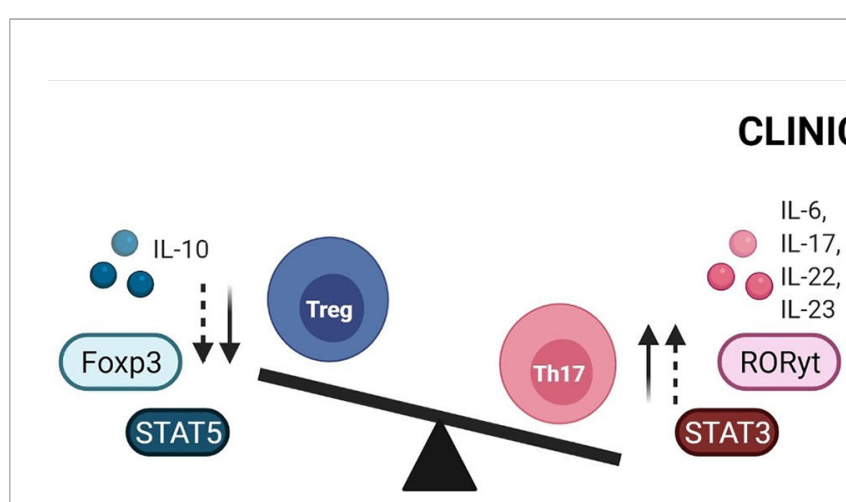

STABLE COPD

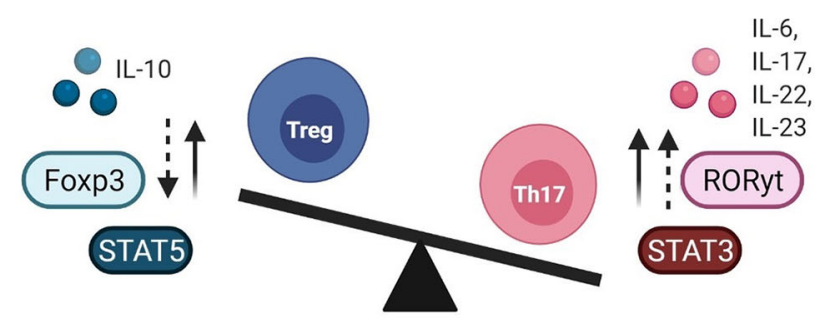

COPD EXACERBATION

\section{EXPERIMENTAL STUDIES}

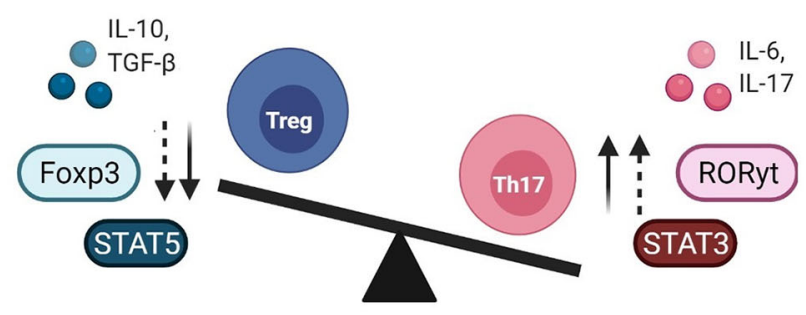

\section{ACUTE AND CHRONIC CS EXPOSED MICE}
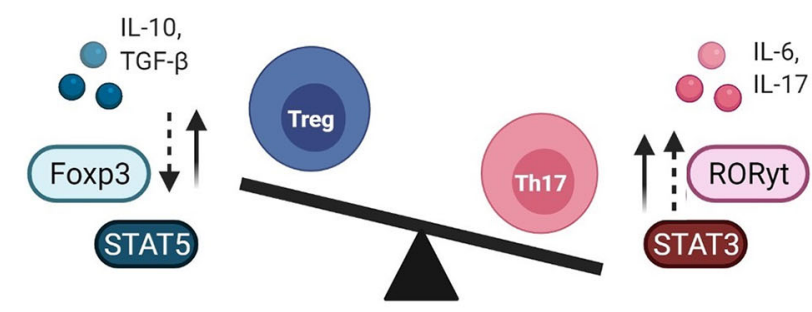

\section{CS + LPS OR CS + NTHi EXPOSED MICE}

- Decreased cell \begin{tabular}{l|l} 
function and & Decreased cell \\
cytokine release & amount
\end{tabular} $\downarrow$ cytokine release

\section{Decreased cell} cytokine release
Increased cell

amount

FIGURE 1 | Th17/Treg imbalance in COPD. Representative image of Th17/Treg imbalance in clinical and experimental studies, in both stable and exacerbated disease. An increase in both Th17 cell amount and function is observed in clinical and experimental studies, during stable and exacerbated disease. In contrast, the Treg response may present increased cell amounts, however with decreased function and cytokine release, leading to Th17/Treg imbalance. COPD, chronic obstructive pulmonary disease; Treg, regulatory T cell; Th17, T helper-17, IL, interleukin; Foxp3, Forkhead box p3; STAT, signal transducer and activator of transcription; ROR $\gamma t$, retinoic acid orphan receptor; TGF- $\beta$, transforming growth factor- $\beta$; CS, cigarette smoke; LPS, lipopolysaccharide; NTHi, Haemophilus influenzae. 
Considering that Foxp3-expressing Treg cells are a heterogeneous cell group comprising distinct subpopulations with different phenotypes and functions (84), it is reasonable to think that to better understand the physiological mechanisms in COPD development, it is more important to distinguish these different cell phenotypes instead of the total number of Tregs. However, until now, there have been only a few studies describing these different phenotypes as well as the immunosuppressive activity of these cells in COPD.

There are 3 described subtypes of Treg cells: resting Tregs (rTregs, CD25++CD45RA+), activated Tregs (aTregs, CD25+++ CD45RA-) and cytokine-secreting Tregs (Frlll, CD25++ CD45RA-Frlll), which were demonstrated to be imbalanced in COPD. Resting and activated Treg cells have suppressor activity, while Treg Frlll cells have a proinflammatory capacity and can produce IL-2, IL-17 and interferon- $\gamma($ IFN- $\gamma$ ) (85).

Hou et al. (2013) evaluated the proportion of Treg cell phenotypes (rTregs, aTregs, and Treg-Frlll) in the peripheral blood and BALF of nonobstructive smokers, never-smokers and individuals with COPD in stages II and III (84). Blood samples demonstrated decreased rTreg and aTreg proportions and increased Treg-Frlll in COPD individuals compared to nonobstructive smokers. Similarly, decreased aTreg cells and increased Treg-FrIII levels were demonstrated in the BALF samples of COPD patients coto healthy smokers and neversmokers. In addition, the authors observed a decrease in the (aTreg + rTeg): Treg-Frlll ratio in both blood and BALF. These findings suggest that the imbalance between the antiinflammatory subpopulations (aTreg + rTeg) and the proinflammatory subpopulation (Fr III) of Tregs plays an important role in the progression of COPD (84).

The imbalance between Treg cell subpopulations was also demonstrated in individuals with COPD after exacerbation.

\section{REFERENCES}

1. Global Initiative for Chronic Obstructive Lung Disease (GOLD). Global Strategy for Diagnosis, Management and Prevention of COPD; 2020 Report. (Fontana, WI, USA: Global Initiative for Chronic Obstructive Lung Disease (GOLD)) (2020).

2. WHO. Projections of Mortality and Causes of Death, 2016 to 2060. Geneva, Switzerland: WHO (2018).

3. Barnes PJ. Cellular and Molecular Mechanisms of Chronic Obstructive Pulmonary Disease. Clin Chest Med (2014) 35:71-86. doi: 10.1016/ j.ccm.2013.10.004

4. Agustí A, Edwards LD, Rennard SI, MacNee W, Tal-Singer R, Miller BE, et al. Persistent Systemic Inflammation is Associated With Poor Clinical Outcomes in Copd: A Novel Phenotype. PloS One (2012) 7(5):e37483. doi: 10.1371/ journal.pone. 0037483

5. Brusselle GG, Joos GF, Bracke KR. New Insights Into the Immunology of Chronic Obstructive Pulmonary Disease. Lancet (2011) 378:1015-26. doi: 10.1016/S0140-6736(11)60988-4

6. Cosio MG, Saetta M, Agusti A. Immunologic Aspects of Chronic Obstructive Pulmonary Disease. N Engl J Med (2009) 360(23):2445-54. doi: 10.1056/ NEJMra0804752

7. Duan MC, Huang Y, Zhong XN, Tang HJ. Th17 Cell Enhances CD8 T-Cell Cytotoxicity via IL-21 Production in Emphysema Mice. Mediators Inflamm (2012) 2012:898053. doi: 10.1155/2012/898053
Yang et al. (2017) found an increase in the percentage of Treg cells secreting cytokines and a decrease in resting Treg cells in both patients with stable COPD and individuals with COPD after exacerbation (86). The authors suggested that there is an increased expression of Treg subtypes with low or no suppressive activity in the blood of individuals with COPD, accompanied by decreased expression of Treg subtypes with suppressive activity (86).

Since there is evidence of increased Treg cells with low or no suppressive activity in COPD individuals, additional studies will be necessary to investigate the distribution of these phenotypes in different stages of COPD. The elucidation of these cell functions as well as which physiological mechanisms orchestrated by these cells are involved in COPD progression seems to be essential to better elucidate this respiratory disease development and progression. In addition, this could be a promising pathway to find new therapeutic approaches.

\section{AUTHOR CONTRIBUTIONS}

FD conceived of the manuscript. JL and FD drafted the manuscript. JL and JI created figures and tables. JL, IT, and MM provided literature search and edited the manuscript. All authors were involved in scientific discussion of the review. All authors contributed to the manuscript and approved the submitted version.

\section{FUNDING}

This research was supported by FAPESP (2019/25374-7).
8. Urbanowicz RA, Lamb JR, Todd I, Corne JM, Fairclough LC. Enhanced Effector Function of Cytotoxic Cells in the Induced Sputum of COPD Patients. Respir Res (2010) 11(1):76. doi: 10.1186/1465-9921-11-76

9. Caramori G, Casolari P, Barczyk A, Durham AL, Di Stefano A, Adcock I. COPD Immunopathology. Semin Immunopathol (2016) 38:497-515. doi: 10.1007/s00281-016-0561-5

10. Wang $\mathrm{H}$, Ying $\mathrm{H}$, Wang $\mathrm{S}, \mathrm{Gu} \mathrm{X}$, Weng $\mathrm{Y}$, Peng $\mathrm{W}$, et al. Imbalance of Peripheral Blood Th17 and Treg Responses in Patients With Chronic Obstructive Pulmonary Disease. Clin Respir J (2015) 9:330-41. doi: 10.1111/ crj. 12147

11. Lane N, Robins RA, Corne J, Fairclough L. Regulation in Chronic Obstructive Pulmonary Disease: The Role of Regulatory T-Cells and Th17 Cells. Clin Sci (2010) 119:75-86. doi: 10.1042/CS20100033

12. Lee SH, Goswami S, Grudo A, Song LZ, Bandi V, Goodnight-White S, et al. Antielastin Autoimmunity in Tobacco Smoking-Induced Emphysema. Nat Med (2007) 13(5):567-9. doi: 10.1038/nm1583

13. Janssen R, Piscaer I, Franssen FME, Wouters EFM. Emphysema: Looking Beyond Alpha-1 Antitrypsin Deficiency. Expert Rev Respir Med (2019) 13 (4):381-97. doi: 10.1080/17476348.2019.1580575

14. Pavord ID, Jones PW, Burgel PR, Rabe KF. Exacerbations of COPD. Int J Chron Obstruct Pulmon Dis (2016) 11 Spec Iss(Spec Iss):21-30. doi: 10.2147/ COPD.S85978

15. Di Stefano A, Caramori G, Gnemmi I, Contoli M, Vicari C, Capelli A, et al. T Helper Type 17-Related Cytokine Expression is Increased in the Bronchial 
Mucosa of Stable Chronic Obstructive Pulmonary Disease Patients. Clin Exp Immunol (2009) 157:316-24. doi: 10.1111/j.1365-2249.2009.03965.x

16. Zhang L, Cheng Z, Liu W, Wu K. Expression of Interleukin (IL)-10, IL-17A and IL-22 in Serum and Sputum of Stable Chronic Obstructive Pulmonary Disease Patients. COPD J Chronic Obstr Pulm Dis (2013) 10:459-65. doi: 10.3109/15412555.2013.770456

17. Traves S, Donnelly L. Th17 Cells in Airway Diseases. Curr Mol Med (2008) 8:416-26. doi: 10.2174/156652408785160998

18. Williams LM, Rudensky AY. Maintenance of the Foxp3-Dependent Developmental Program in Mature Regulatory T Cells Requires Continued Expression of Foxp3. Nat Immunol (2007) 8:277-84. doi: 10.1038/ni1437

19. Bedke T, Muscate F, Soukou S, Gagliani N, Huber S. Title: IL-10-Producing T Cells and Their Dual Functions. Semin Immunol (2019) 44:101335. doi: 10.1016/j.smim.2019.101335

20. Saraiva M, Saraiva M, Vieira P, Vieira P, Vieira P, O'Garra A, et al. Biology and Therapeutic Potential of Interleukin-10. J Exp Med (2020) 217:1-19. doi: 10.1084/jem_20190418

21. Elizondo DM, Andargie TE, Haddock NL, da Silva RLL, de Moura TR, Lipscomb MW. IL-10 Producing CD8 + CD122 + PD-1 + Regulatory T Cells are Expanded by Dendritic Cells Silenced for Allograft Inflammatory Factor1. J Leukoc Biol (2019) 105:123-30. doi: 10.1002/JLB.1A0118-010RR

22. Yao Y, Simard AR, Shi FD, Hao J. IL-10-Producing Lymphocytes in Inflammatory Disease. Int Rev Immunol (2013) 32:324-36. doi: 10.3109/ 08830185.2012.762361

23. Ouyang W, O'Garra A. IL-10 Family Cytokines IL-10 and IL-22: From Basic Science to Clinical Translation. Immunity (2019) 50:871-91. doi: 10.1016/ j.immuni.2019.03.020

24. Chaudhry A, Samstein RM, Treuting P, Liang Y, Pils MC, Heinrich JM, et al. Interleukin-10 Signaling in Regulatory T Cells Is Required for Suppression of Th17 Cell-Mediated Inflammation. Immunity (2011) 34:566-78. doi: 10.1016/ j.immuni.2011.03.018

25. Ito JT, De Brito Cervilha DA, Lourenço JD, Gonçalves NG, Volpini RA, Caldini EG, et al. Th17/Treg Imbalance in COPD Progression: A Temporal Analysis Using a CS-Induced Model. PloS One (2019) 14(1):e0209351. doi: 10.1371/journal.pone.0209351

26. Silva LEF, Lourenço JD, Silva KR, Santana FPR, Kohler JB, Moreira AR, et al. Th17/Treg Imbalance in COPD Development: Suppressors of Cytokine Signaling and Signal Transducers and Activators of Transcription Proteins. Sci Rep (2020) 10(1):15287. doi: 10.1038/s41598-020-72305-y

27. Lourenço JD, Teodoro WR, Barbeiro DF, Velosa APP, Silva LEF, Kohler JB, et al. Th17/Treg-Related Intracellular Signaling in Patients With Chronic Obstructive Pulmonary Disease: Comparison Between Local and Systemic Responses. Cells (2021) 10(7):1569. doi: 10.3390/cells10071569

28. Jin Y, Wan Y, Chen G, Chen L, Zhang MQ, Deng L, et al. Treg/IL-17 Ratio and Treg Differentiation in Patients With COPD. PloS One (2014) 9(10):e111044. doi: 10.1371/journal.pone.0111044

29. Chu S, Zhong X, Zhang J, Lao Q, He Z, Bai J. The Expression of Foxp3 and ROR Gamma $\mathrm{T}$ in Lung Tissues From Normal Smokers and Chronic Obstructive Pulmonary Disease Patients. Int Immunopharmacol (2011) 11:1780-8. doi: 10.1016/j.intimp.2011.06.010

30. Li XN, Pan X, Qiu D. Imbalances of Th17 and Treg Cells and Their Respective Cytokines in COPD Patients by Disease Stage. Int J Clin Exp Med (2014) 7:5324-9.

31. Silva BSA, Lira FS, Ramos D, Uzeloto JS, Rossi FE, Freire APCF, et al. Severity of COPD and its Relationship With IL-10. Cytokine (2018) 106:95-100. doi: 10.1016/j.cyto.2017.10.018

32. Wang H, Peng W, Weng Y, Ying H, Li H, Xia D, et al. Imbalance of Th17/Treg Cells in Mice With Chronic Cigarette Smoke Exposure. Int Immunopharmacol (2012) 14(4):504-12. doi: 10.1016/j.intimp.2012.09.011

33. Duan MC, Zhang JQ, Liang Y, Liu GN, Xiao J, Tang HJ, et al. Infiltration of IL17-Producing T Cells and Treg Cells in a Mouse Model of Smoke-Induced Emphysema. Inflammation (2016) 39(4):1334-44. doi: 10.1007/s10753-0160365-8

34. Guan X, Lu Y, Wang G, Gibson P, Chen F, Fang K, et al. The Role of Regulatory T Cell in Nontypeable Haemophilus Influenzae-Induced Acute Exacerbation of Chronic Obstructive Pulmonary Disease. Mediators Inflammation (2018) 2018:8387150. doi: 10.1155/2018/8387150
35. Cervilha DAB, Ito JT, Lourenço JD, Olivo CR, Saraiva-Romanholo BM, Volpini RA, et al. The Th17/Treg Cytokine Imbalance in Chronic Obstructive Pulmonary Disease Exacerbation in an Animal Model of Cigarette Smoke Exposure and Lipopolysaccharide Challenge Association. Sci Rep (2019) 9(1):1921. doi: 10.1038/s41598-019-38600-z

36. Alcorn JF, Crowe CR, Kolls JK. TH17 Cells in Asthma and COPD. Annu Rev Physiol (2009) 72:495-516. doi: 10.1146/annurev-physiol-021909-135926

37. Roos AB, Sethi S, Nikota J, Wrona CT, Dorrington MG, Sandén C, et al. IL$17 \mathrm{~A}$ and the Promotion of Neutrophilia in Acute Exacerbation of Chronic Obstructive Pulmonary Disease. Am J Respir Crit Care Med (2015) 192 (4):428-37. doi: 10.1164/rccm.201409-1689OC

38. Sileikiene V, Laurinaviciene A, Lesciute-Krilaviciene D, Jurgauskiene L, Malickaite R, Laurinavicius A. Levels of CD4+ CD25+ T Regulatory Cells in Bronchial Mucosa and Peripheral Blood of Chronic Obstructive Pulmonary Disease Indicate Involvement of Autoimmunity Mechanisms. Adv Respir Med (2019) 87:159-66. doi: 10.5603/ARM.2019.0023

39. Smyth LJC, Starkey C, Vestbo J, Singh D. CD4-Regulatory Cells in COPD Patients. Chest (2007) 132(1):156-63. doi: 10.1378/chest.07-0083

40. Eriksson Ström J, Pourazar J, Linder R, Blomberg A, Lindberg A, Bucht A, et al. Airway Regulatory T Cells are Decreased in COPD With a Rapid Decline in Lung Function. Respir Res (2020) 21(1):330. doi: 10.1186/s12931-02001593-9

41. Zheng X, Zhang L, Chen J, Gu Y, Xu J, Ouyang Y. Dendritic Cells and Th17/ Treg Ratio Play Critical Roles in Pathogenic Process of Chronic Obstructive Pulmonary Disease. BioMed Pharmacother (2018) 108:1141-51. doi: 10.1016/ j.biopha.2018.09.113

42. Sales DS, Ito JT, Zanchetta IA, Annoni R, Aun MV, Ferraz LFS, et al. Regulatory TCell Distribution Within Lung Compartments in COPD. COPD J Chronic Obstr Pulm Dis (2017) 14:533-42. doi: 10.1080/15412555.2017.1346069

43. Plumb J, Smyth LJC, Adams HR, Vestbo J, Bentley A, Singh SD. Increased TRegulatory Cells Within Lymphocyte Follicles in Moderate COPD. Eur Respir $J$ (2009) 34:89-94. doi: 10.1183/09031936.00100708

44. Isajevs S, Taivans I, Strazda G, Kopeika U, Bukovskis M, Gordjusina V, et al. Decreased FOXP3 Expression in Small Airways of Smokers With COPD. Eur Respir J (2009) 33(1):61-7. doi: 10.1183/09031936.00145307

45. Upham JW, Xi Y. Dendritic Cells in Human Lung Disease: Recent Advances. Chest (2017) 151:668-73. doi: 10.1016/j.chest.2016.09.030

46. Shan M, Cheng HF, Song LZ, Roberts L, Green L, Hacken-Bitar J, et al. Lung Myeloid Dendritic Cells Coordinately Induce TH1 and T H17 Responses in Human Emphysema. Sci Transl Med (2009) 1:1-11. doi: 10.1126/scitranlsmed.3000154

47. Seif F, Khoshmirsafa M, Aazami H, Mohsenzadegan M, Sedighi G, Bahar M. The Role of JAK-STAT Signaling Pathway and Its Regulators in the Fate of T Helper Cells. Cell Commun Signal (2017) 15:1-13. doi: 10.1186/s12964-017$0177-y$

48. Burchill MA, Yang J, Vogtenhuber C, Blazar BR, Farrar MA. IL-2 Receptor $\beta$ Dependent STAT5 Activation Is Required for the Development of Foxp3 + Regulatory T Cells. J Immunol (2007) 178:280-90. doi: 10.4049/ jimmunol.178.1.280

49. Chen Z, Lin F, Gao Y, Li Z, Zhang J, Xing Y, et al. FOXP3 and Roryt: Transcriptional Regulation of Treg and Th17. Int Immunopharmacol (2011) 11:536-42. doi: 10.1016/j.intimp.2010.11.008

50. Yew-Booth L, Birrell MA, Lau MS, Baker K, Jones V, Kilty I, et al. JAK-STAT Pathway Activation in COPD. Eur Respir J (2015) 46:843-5. doi: 10.1183/ 09031936.00228414

51. Knosp CA, Johnston JA. Regulation of CD4+ T-Cell Polarization by Suppressor of Cytokine Signalling Proteins. Immunology (2012) 135(2):10111. doi: 10.1111/j.1365-2567.2011.03520.x

52. Yamamoto K, Yamaguchi M, Miyasaka N, Miura O. SOCS-3 Inhibits IL-12Induced STAT4 Activation by Binding Through its SH2 Domain to the STAT4 Docking Site in the IL-12 Receptor $\beta 2$ Subunit. Biochem Biophys Res Commun (2003) 310(4):1188-93. doi: 10.1016/j.bbrc.2003.09.140

53. Springer J, Scholz FR, Peiser C, Dinh QT, Fischer A, Quarcoo D, et al. Transcriptional Down-Regulation of Suppressor of Cytokine Signaling (SOCS)-3 in Chronic Obstructive Pulmonary Disease. J Occup Med Toxicol (2013) 8:1. doi: 10.1186/1745-6673-8-29

54. Takahashi R, Nishimoto S, Muto G, Sekiya T, Tamiya T, Kimura A, et al. SOCS1 Is Essential for Regulatory T Cell Functions by Preventing Loss of 
Foxp3 Expression as Well as IFN- $\gamma$ and IL-17A Production. J Exp Med (2011) 208:2055-67. doi: 10.1084/jem.20110428

55. Dong R, Xie L, Zhao K, Zhou M, He P. Cigarette Smoke-Induced Lung Inflammation in COPD Mediated via LTB4/BLT1/SOCS1 Pathway. Int $J$ COPD (2015) 11:31-41. doi: 10.2147/COPD.S96412

56. Barnes PJ. Inflammatory Mechanisms in Patients With Chronic Obstructive Pulmonary Disease. J Allergy Clin Immunol (2016) 138:16-27. doi: 10.1016/ j.jaci.2016.05.011

57. Agustí A. Systemic Effects of Chronic Obstructive Pulmonary Disease: What We Know and What We Don't Know (But Should). Proc Am Thorac Soc (2007) 4(7):522-5. doi: 10.1513/pats.200701-004FM

58. Barnes PJ, Celli BR. Systemic Manifestations and Comorbidities of COPD. Eur Respir J (2009) 33:1165-85. doi: 10.1183/09031936.00128008

59. Vargas-Rojas MI, Ramírez-Venegas A, Limón-Camacho L, Ochoa L, Hernández-Zenteno R, Sansores RH. Increase of Th17 Cells in Peripheral Blood of Patients With Chronic Obstructive Pulmonary Disease. Respir Med (2011) 105(11):1648-54. doi: 10.1016/j.rmed.2011.05.017

60. Xu W, Li R, Sun Y. Increased IFN- $\gamma$-Producing Th17/Th1 Cells and Their Association With Lung Function and Current Smoking Status in Patients With Chronic Obstructive Pulmonary Disease. BMC Pulm Med (2019) 19:19. doi: 10.1186/s12890-019-0899-2

61. Barceló B, Pons J, Ferrer JM, Sauleda J, Fuster A, Agustí AGN. Phenotypic Characterisation of T-Lymphocytes in COPD: Abnormal CD4+CD25+ Regulatory T-Lymphocyte Response to Tobacco Smoking. Eur Respir J (2008) 31:555-62. doi: 10.1183/09031936.00010407

62. Zhou H, Hua W, Jin Y, Zhang C, Che L, Xia L, et al. Tc17 Cells are Associated With Cigarette Smoke-Induced Lung Inflammation and Emphysema. Respirology (2015) 20(3):426-33. doi: 10.1111/resp.12486

63. Duan MC, Tang HJ, Zhong XN, Huang Y. Persistence of Th17/Tc17 Cell Expression Upon Smoking Cessation in Mice With Cigarette Smoke-Induced Emphysema. Clin Dev Immunol (2013) 2013:350727. doi: 10.1155/2013/350727

64. Ruwanpura SM, McLeod L, Brooks GD, Bozinovski S, Vlahos R, Longano A, et al. IL-6/Stat3-Driven Pulmonary Inflammation, But Not Emphysema, is Dependent on Interleukin-17A in Mice. Respirology (2014) 19:419-27. doi: $10.1111 /$ resp. 12243

65. Hogg JC, Chu F, Utokaparch S, Woods R, Elliott WM, Buzatu L, et al. The Nature of Small-Airway Obstruction in Chronic Obstructive Pulmonary Disease. N Engl J Med (2004) 350(26):2645-53. doi: 10.1056/nejmoa032158

66. Pabst R, Tschernig T. Perivascular Capillaries in the Lung: An Important But Neglected Vascular Bed in Immune Reactions? J Allergy Clin Immunol (2002) 110(2):209-14. doi: 10.1067/mai.2002.126836

67. Barnes NC, Qiu YS, Pavord ID, Parker D, Davis PA, Zhu J, et al. Antiinflammatory Effects of Salmeterol/Fluticasone Propionate in Chronic Obstructive Lung Disease. Am J Respir Crit Care Med (2006) 173(7):736-43. doi: $10.1164 /$ rccm.200508-1321OC

68. Brusselle G, Bracke K. Targeting Immune Pathways for Therapy in Asthma and Chronic Obstructive Pulmonary Disease. Ann Am Thorac Soc (2014) 11 (Suppl 5):S322-8. doi: 10.1513/AnnalsATS.201403-118AW

69. Barnes PJ. New Anti-Inflammatory Targets for Chronic Obstructive Pulmonary Disease. Nat Rev Drug Discov (2013) 12(7):543-59. doi: 10.1038/nrd4025

70. Profita M, Albano GD, Riccobono L, Di Sano C, Montalbano AM, Gagliardo $\mathrm{R}$, et al. Increased Levels of Th17 Cells are Associated With Non-Neuronal Acetylcholine in COPD Patients. Immunobiology (2014) 219(5):392-401. doi: 10.1016/j.imbio.2014.01.004

71. Tan C, Huang H, Zhang J, He Z, Zhong X, Bai J. Effects of Low-Dose and LongTerm Treatment With Erythromycin on IL-17, IL-23 in Peripheral Blood and Induced Sputum in Patients With Stable Chronic Obstructive Pulmonary Disease. Mediators Inflamm (2016) 2016:4173962. doi: 10.1155/2016/4173962

72. Tang S, Tingting M, Zhang H, Zhang J, Zhong X, Tan C, et al. Erythromycin Prevents Elastin Peptide-Induced Emphysema and Modulates CD4+T Cell Responses in Mice. Int J COPD (2019) 14:2697-709. doi: 10.2147/COPD.S222195

73. Liu J, Zhong X, He Z, Zhang J, Bai J, Liu G, et al. Erythromycin Suppresses the Cigarette Smoke Extract-Exposed Dendritic Cell-Mediated Polarization of CD4+ T Cells Into Th17 Cells. J Immunol Res (2020) 2020:1387952. doi: $10.1155 / 2020 / 1387952$

74. Liu X, Hu Z, Zhou H. N-Acetylcysteine Improves Inflammatory Response in COPD Patients by Regulating Th17/Treg Balance Through Hypoxia Inducible
Factor-1 $\alpha$ Pathway. BioMed Res Int (2021) 2021:6372128. doi: 10.1155/2021/ 6372128

75. Barbi J, Pardoll D, Pan F. Metabolic Control of the Treg/Th17 Axis. Immunol $\operatorname{Rev}(2013)$ 252:52-77. doi: 10.1111/imr.12029

76. dos Santos TM, Righetti RF, Camargo L do N, Saraiva-Romanholo BM, Aristoteles LRCRB, de Souza FCR, et al. Effect of Anti-IL17 Antibody Treatment Alone and in Combination With Rho-Kinase Inhibitor in a Murine Model of Asthma. Front Physiol (2018) 9:1183. doi: 10.3389/ fphys.2018.01183

77. dos Santos TM, Righetti RF, Rezende BG, Campos EC, Camargo L do N, Saraiva-Romanholo BM, et al. Effect of Anti-IL17 and/or Rho-Kinase Inhibitor Treatments on Vascular Remodeling Induced by Chronic Allergic Pulmonary Inflammation. Ther Adv Respir Dis (2020) 14:1753466620962665. doi: $10.1177 / 1753466620962665$

78. Camargo L do N, Righetti RF, Aristóteles LR de CRB, dos Santos TM, de Souza FCR, Fukuzaki S, et al. Effects of Anti-IL-17 on Inflammation, Remodeling, and Oxidative Stress in an Experimental Model of Asthma Exacerbated by LPS. Front Immunol (2018) 8:1835. doi: 10.3389/ fimmu.2017.01835

79. Camargo L do N, dos Santos TM, de Andrade FCP, Fukuzaki S, dos Santos Lopes FDTQ, de Arruda Martins M, et al. Bronchial Vascular Remodeling Is Attenuated by Anti-IL-17 in Asthmatic Responses Exacerbated by LPS. Front Pharmacol (2020) 11:1269. doi: 10.3389/fphar.2020.01269

80. Righetti RF, Dos Santos TM, Camargo LDN, Barbosa Aristóteles LRCR, Fukuzaki S, De Souza FCR, et al. Protective Effects of Anti-IL17 on Acute Lung Injury Induced by LPS in Mice. Front Pharmacol (2018) 9:1021. doi: $10.3389 /$ fphar.2018.01021

81. Fukuzaki S, Righetti RF, dos Santos TM, do Nascimento Camargo L, Aristóteles LRCRB, Souza FCR, et al. Preventive and Therapeutic Effect of Anti-IL-17 in an Experimental Model of Elastase-Induced Lung Injury in C57Bl6 Mice. Am J Physiol - Cell Physiol (2021) 320(3):C341-54. doi: 10.1152/ ajpcell.00017.2020

82. Nasreen N, Gonzalves L, Peruvemba S, Mohammed KA. Fluticasone Furoate is More Effective Than Mometasone Furoate in Restoring Tobacco Smoke Inhibited SOCS-3 Expression in Airway Epithelial Cells. Int Immunopharmacol (2014) 19:153-60. doi: 10.1016/j.intimp.2013.12.029

83. Chiappori A, Folli C, Balbi F, Caci E, Riccio AM, De Ferrari L, et al. CD4 +CD25highCD127-Regulatory T-Cells in COPD: Smoke and Drugs Effect. World Allergy Organ J (2016) 9:5. doi: 10.1186/s40413-016-0095-2

84. Hou J, Sun Y, Hao Y, Zhuo J, Liu X, Bai P, et al. Imbalance Between Subpopulations of Regulatory T Cells in COPD. Thorax (2013) 68:1131-9. doi: 10.1136/thoraxjnl-2012-201956

85. Miyara M, Yoshioka Y, Kitoh A, Shima T, Wing K, Niwa A, et al. Functional Delineation and Differentiation Dynamics of Human CD4+ T Cells Expressing the FoxP3 Transcription Factor. Immunity (2009) 30(6):899911. doi: 10.1016/j.immuni.2009.03.019

86. Yang X, Huo B, Zhong X, Su W, Liu W, Li Y, et al. Imbalance Between Subpopulations of Regulatory T Cells in Patients With Acute Exacerbation of COPD. COPD J Chronic Obstr Pulm Dis (2017) 14(6):618-25. doi: 10.1080/ 15412555.2017.1385055

Conflict of Interest: The authors declare that the research was conducted in the absence of any commercial or financial relationships that could be construed as a potential conflict of interest.

Publisher's Note: All claims expressed in this article are solely those of the authors and do not necessarily represent those of their affiliated organizations, or those of the publisher, the editors and the reviewers. Any product that may be evaluated in this article, or claim that may be made by its manufacturer, is not guaranteed or endorsed by the publisher.

Copyright $\odot 2021$ Lourenço, Ito, Martins, Tibério and Lopes. This is an open-access article distributed under the terms of the Creative Commons Attribution License (CC BY). The use, distribution or reproduction in other forums is permitted, provided the original author(s) and the copyright owner(s) are credited and that the original publication in this journal is cited, in accordance with accepted academic practice. No use, distribution or reproduction is permitted which does not comply with these terms. 